\author{
The AFP and PPS projects \\ Christophe Royon \\ IRFU-SPP, CEA Saclay, F91191 Gif-sur-Yvette cedex \\ Nicolo Cartiglia \\ INFN Torino, Via Pietro Giuria 1, Torino, Italy
}

\begin{abstract}
We present the project to install new forward proton detectors in the CMS and ATLAS experiments called PPS and AFP respectively.

PACS numbers: 13.85.-t,11.25.Wx, 12.15.-y, 12.38.-t
\end{abstract}

\title{
1. The AFP and CT-PPS projects
}

The Large Hadron Collider (LHC) will collide protons with a center-ofmass energy of $13 \mathrm{TeV}$ starting in 2015. Several improvements are made to the ATLAS and CMS detectors to exploit this new energy regime; this short report describes the project to install the ATLAS Forward Proton (AFP) detector at 206 and 214 meters on both sides of the ATLAS experiment [3] (see Fig. 11) and the similar project by the TOTEM and CMS collaborations, the so called CT-PPS, to be installed on both sides of the CMS detector. In this article, we will concentrate on the main characteristics of the AFP and CT-PPS detectors, while their physics reach is described elsewhere [1, 2]..

Each arm of the AFP detector will consist of two sections: AFP1 at 206 meters, and AFP2, at 220 meters. In AFP1, a tracking station composed by 6 layers of Silicon detectors will be deployed. The second section, AFP2, will contain a second identical tracking station and a timing detector. In addition, a similar structure could be installed at about $420 \mathrm{~m}$ from the ATLAS interaction point. The aim of the combined two arms of this setup is to tag the protons emerging intact from the pp interactions, allowing ATLAS to exploit the program of diffractive and photon induced processes described in the previous sections. Likewise, the CT-PPS of CMS will also use the same combination of tracking and timing detectors, with the far station using specially designed cylindrical roman pots to house the timing detectors. 


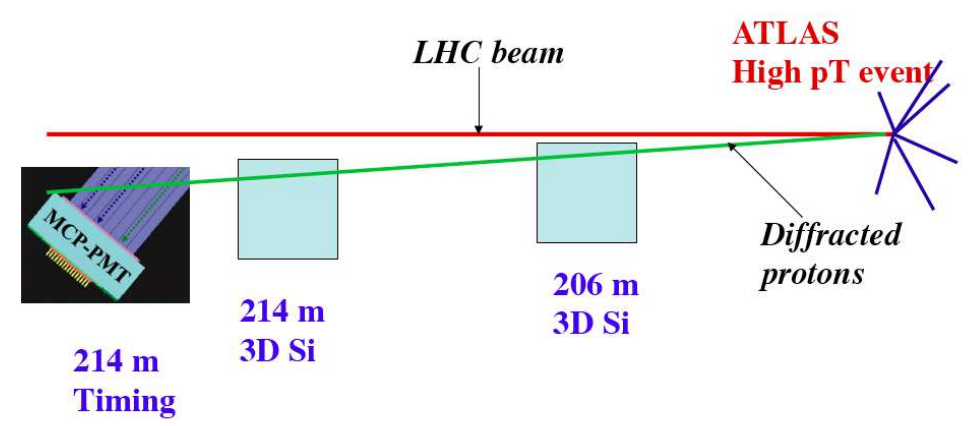

Fig. 1. Scheme of the AFP proton detector in ATLAS. The same detector is implemented on the other side of ATLAS.

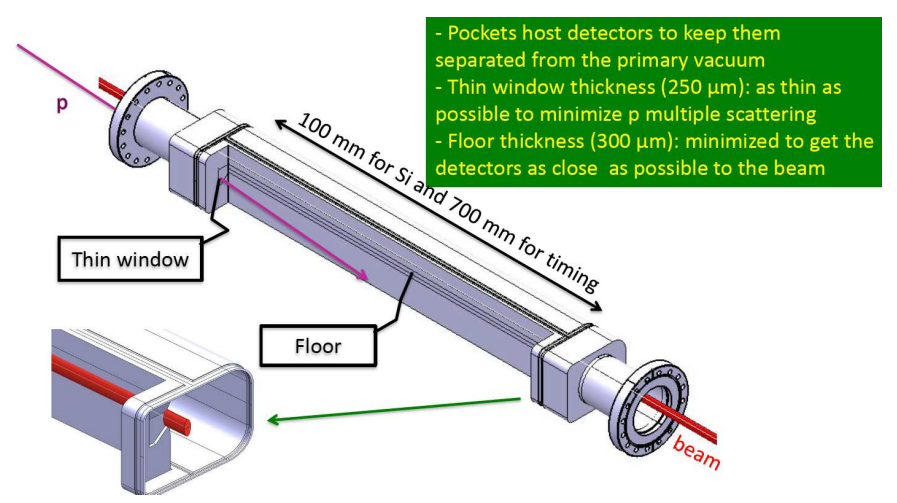

Fig. 2. Scheme of the movable beam pipe.

\section{Movable beam pipes and roman pots}

In order to house the detectors needed by the AFP and CT-PPS projects, two different types of modification of the beam-pipe are currently considered: (i) roman pots and (ii) movable beam pipes). Roman pots have been used already in many experiments at the SPS, HERA, Tevatron and LHC colliders (in the TOTEM and ATLAS-ALFA experiments). The roman pots, in their basic design, are pockets where the detectors can be hosted. These pockets are pushed inside the beam pipe to a position closer to the beam line once stable beam has been declared (a typical motion is of the order of a few $\mathrm{cm}$ ). To minimize multiple scattering, protons will enter the roman pots via a thin window located at the bottom of the pot, on the side facing the beams. Different types of roman pots can host the tracking and timing detectors: tracking detectors need less space than timing detectors, and therefore can be housed in smaller roman pots. . Conversely, in the movable 
beam pipe design, no pocket is pushed closer to the beam, but the whole beam pipe moves closer to the beam. The idea of movable Hamburg beam pipes is quite simple [4]: a section of the LHC beam pipe is replaced by a larger one, specially designed to have a cutout to host the detectors. When allowed by beam conditions, using specially designed bellows that allow for transverse motions, this part of the beam pipe is moved, by about $2.5 \mathrm{~cm}$, so that the detectors located at its edge (called pocket) are brought closer to the beam. In its design, the most challenging aspect is the minimization of the thickness of the portions called floor and window (see Fig. 2). This is necessary as the floor might be rather long, of the order of 10-40 centimeters in the direction parallel to the motion of the particles: minimizing its depth of the floor ensures that the detectors can be brought as close to the beam as possible allowing detecting protons scattered at very small angles. Two configurations exist for the movable beam pipes: the first one at $206 \mathrm{~m}$ from the ATLAS interaction point hosts a Si detector (floor length of about 100 $\mathrm{mm}$ ) and the second one (floor length of about $400 \mathrm{~mm}$ ) the timing and the Si detectors.

The AFP and CT-PPS detectors will use Roman Pots in their starting configuration. In the meantime, the development of the Hamburg beam pipe is carried on together by both collaborations. However, it is clear that movable beam pipes are needed at $420 \mathrm{~m}$, if later upgrades include installation of forward detectors at that location. At $420 \mathrm{~m}$, not enough space is available and new specially designed cryostats have been developed to host these movable beam pipes in the cold region. The usage of roman pots at $420 \mathrm{~m}$ would require a costly cryogenic bypass to be installed to isolate the part of the beam pipe where roman pots would be installed.

\section{3D Silicon detectors}

The purpose of the tracker system is to measure points along the trajectory of beam protons that are deflected at small angles as a result of collisions. The tracker, when combined with the LHC dipole and quadrupole magnets, forms a powerful momentum spectrometer. Silicon tracker stations will be installed in Hamburg beam pipes or roman pots at \pm 206 and $\pm 214 \mathrm{~m}$ from the ATLAS interaction point (and also at $420 \mathrm{~m}$ later if these additional detectors are appoved).

The key requirements for the silicon tracking system at $220 \mathrm{~m}$ are:

- Spatial resolution of $\sim 10$ (30) $\mu$ m per detector station in $x(y)$

- Angular resolution for a pair of detectors of a few $\mu \mathrm{rad}$

- High efficiency over the area of $20 \mathrm{~mm} \times 20 \mathrm{~mm}$ corresponding to the distribution of diffracted protons 
- Minimal dead space at the edge of the sensors towards the beam line, allowing measuring the scattered protons at low angles

- Sufficient radiation hardness in order to sustain the radiation at high luminosity

- Capable of robust and reliable operation at high LHC luminosity

The basic building unit of the AFP detection system is a module consisting of an assembly of a sensor array, on-sensor read-out chip(s), electrical services, data acquisition and detector control system. The module will be mounted on the mechanical support with embedded cooling and other necessary services. The sensors are double sided 3D $50 \times 250$ micron pixel detectors with slim-edge dicing built by FBK and CNM companies. The sensor efficiency has been measured to be close to $100 \%$ over the full size in beam tests. A possible upgrade of this device will be to use 3D edgeless Silicon detectors built in a collaboration between SLAC, Manchester, Oslo, Bergen...

A new front-end chip FE-I4 has been developed for the Si detector by the Insertable B Layer (IBL) collaboration in ATLAS [5]. The FE-I4 integrated circuit contains readout circuitry for 26880 hybrid pixels arranged in 80 columns on $250 \mu \mathrm{m}$ pitch by 336 rows on $50 \mu \mathrm{m}$ pitch, and covers an area of about $19 \mathrm{~mm} \times 20 \mathrm{~mm}$. It is designed in a $130 \mathrm{~nm}$ feature size bulk CMOS process. Sensors must be DC coupled to FE-I4 with negative charge collection. The FE-I4 is very well suited to the AFP requirements: the granularity of cells provides a sufficient spatial resolution, the chip is radiation hard enough up to a dose of $3 \mathrm{MGy}$, and the size of the chip is sufficiently large that one module can be served by just one chip.

The dimensions of the individual cells in the FE-I4 chip are $50 \mu \mathrm{m} \times 250$ $\mu \mathrm{m}$ in the $x$ and $y$ directions, respectively. Therefore to achieve the required position resolution in the $x$-direction of $\sim 10 \mu \mathrm{m}$, six layers with sensors are required (this gives $50 / \sqrt{12} / \sqrt{5} \sim 7 \mu \mathrm{m}$ in $x$ and roughly 5 times worse in $y$ ). Offsetting planes alternately to the left and right by one half pixel will give a further reduction in resolution of at least $30 \%$. The AFP sensors are expected to be exposed to a dose of $30 \mathrm{kGy}$ per year at the full LHC luminosity of $10^{34} \mathrm{~cm}^{-2} \mathrm{~s}^{-1}$.

The baseline CT-PPS tracking system is also based on 3D pixel sensors, produced either by FBK (Trento, Italy) or CNM (Barcelona, Spain), which provide the best performance in terms of active region and radiation hardness.

The chosen configuration for the tracking system consists of two detector stations in each arm. Each station will contain one stack of silicon tracking detectors. Each stack will consist of six planes, where each plane conatains a 


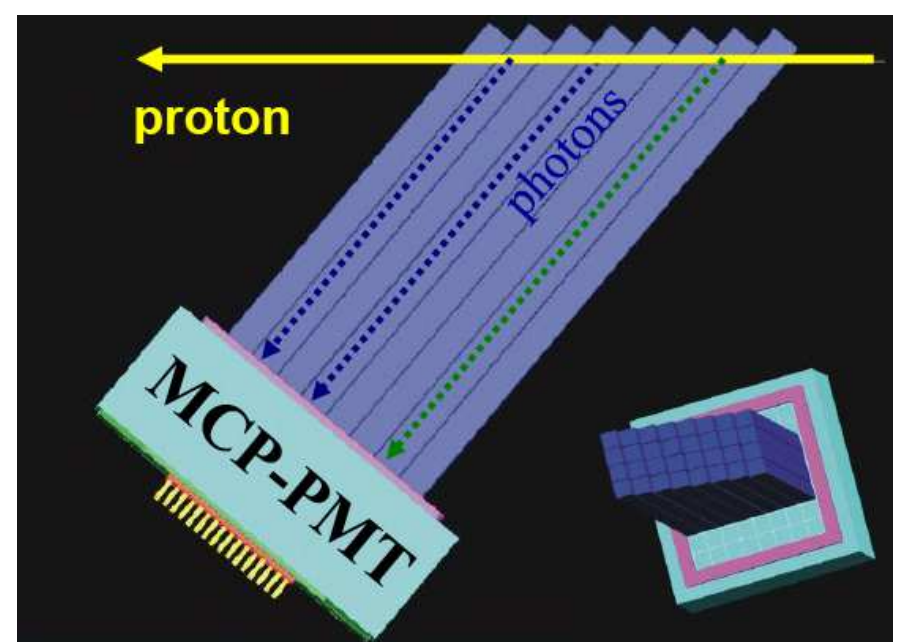

Fig. 3. A schematic diagram of the QUARTIC fast timing detector.

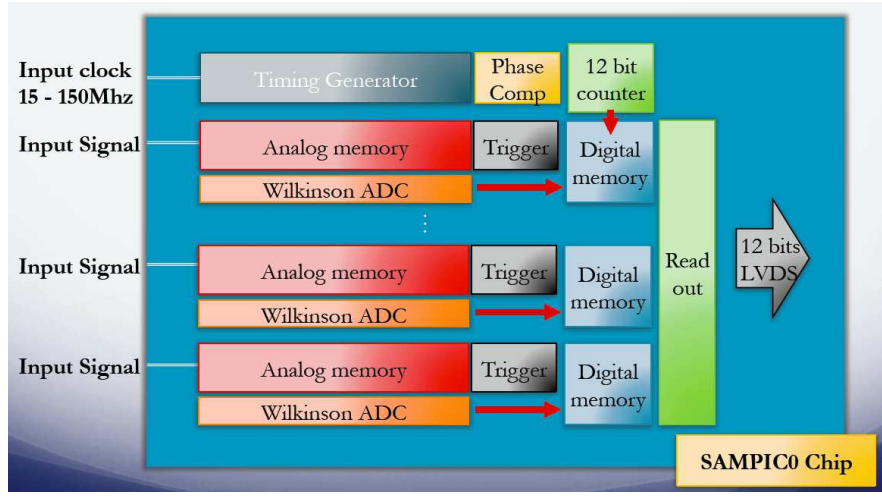

Fig. 4. Principle of the sampic chip.

$1.6 \times 2.4 \mathrm{~cm}^{2}$ pixel sensor read out by six PSI46dig readout chips ROCs 6 . Each ROC reads $52 \times 80$ pixels with dimensions $150 \times 100 \mu \mathrm{m}^{2}$. The design of the front-end electronics and of the DAQ is based on that developed for the Phse I upgrade of the CMS silicon pixel detectors [7.

\section{Timing detectors}

\subsection{Requirements and present achievement}

A fast timing system that can precisely measure the time difference between the two outgoing scattered protons is a key component of the AFP and CT-PPS detectors. The time difference is equivalent to a constraint on the event vertex, thus the AFP and CT-PPS timing detectors can be 


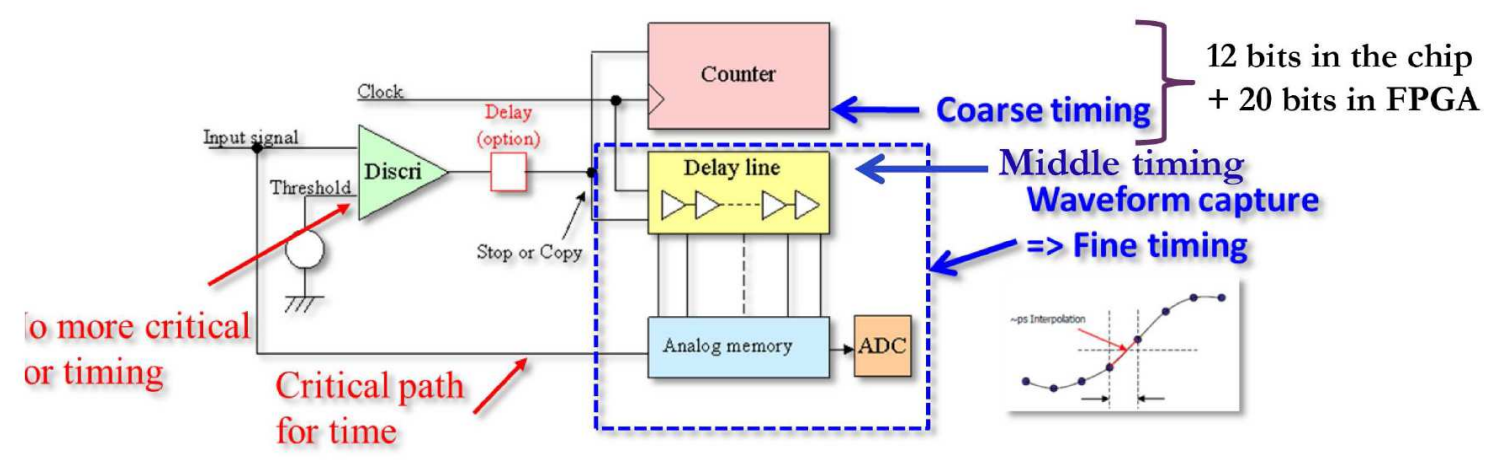

Fig. 5. Scheme of the sampic chip.

used to reject overlapping background events by establishing that the two scattered protons did not originate from the same vertex that triggered the central ATLAS or CMS detectors. The final timing system should have the following characteristics [8]:

- 10 ps or better resolution (which leads to a factor 40 rejection on pile up background)

- Efficiency close to $100 \%$ over the full detector coverage

- High rate capability (there is a bunch crossing every 25 ns at the nominal LHC)

- Enough segmentation for multi-proton timing

- High level trigger capability

Fig. 3 shows a schematic overview of the first proposed timing system in AFP, consisting of a quartz-based Cerenkov detector coupled to a microchannel plate photomultiplier tube (MCP-PMT), followed by the electronic elements that amplify, measure, and record the time of the event along with a stabilized reference clock signal. The QUARTIC detector consists of an array of $8 \times 4$ fused silica bars ranging in length from about 8 to $12 \mathrm{~cm}$ and oriented at the average Cerenkov angle. A proton that is sufficiently deflected from the beam axis will pass through a row of eight bars emitting Cerenkov photons providing an overall time resolution that is approximately $\sqrt{8}$ times smaller than the single bar resolution of about 30 ps, thus approaching the $10 \mathrm{ps}$ resolution goal. Prototype tests have generally been performed on one row ( 8 channels) of $5 \mathrm{~mm} \times 5 \mathrm{~mm}$ pixels, while 
the initial detector is foreseen to have four rows to obtain full acceptance out to $20 \mathrm{~mm}$ from the beam. The beam tests lead to a time resolution per bar of the order of $34 \mathrm{ps}$. The upgraded design of the timing detector has equal rate pixels, and we plan to reduce the width of detector bins close to the beam, where the proton density is highest.

The CT-PPS also has a detector based on Cerenkov technology as the baseline proposal. It has chosen a Cherenkov L-bar Quartic design (Quartz Timing Cherenkov) with $5 \times 4$ equal to twenty $3 \times 3 \mathrm{~mm}^{2}$ independent channels. They are read-out by SiPM photodetectors, relatively far from the beam, in a region where the neutron flux is $\sim 210^{12} \mathrm{neq} / \mathrm{cm}^{2}$ per $100 \mathrm{fb}^{-1}$. SiPM devices that tolerate this radiation level are available, and already in use in the CMS detector [9. The SiPMs will probably require replacement after $100 \mathrm{fb}^{-1}$, which is feasible given the small number of devides involved. Two such Quartic detectors fit inside a cylindrical roman pot, providing a combined resolution of the order of $20 \mathrm{ps}$.

\subsection{Future developments}

At higher luminosity of the LHC (phase I starting in 2019), higher pixelisation of the timing detector will be required in order to fight against high pile up environment [12]. For this sake, a R\&D phase to develop timing detector developments based on Silicon sensors 10, and diamonds 11 has started. This new R\&D aims at installing a prototype of such detector at the LHC in the TOTEM experiment as soon as they are available. In parallel to this sensor $\mathrm{R} \& \mathrm{D}$, a new timing readout chip has been developed in Saclay. It uses waveform sampling to reach the best possible timing resolution: single-threshold and multi-threshold circuits are much more affected by the negative effects of time walk and jitter on the time resolution.. The

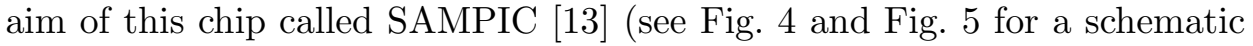
view of SAMPIC) is to obtain sub 10 ps timing resolution, $1 \mathrm{GHz}$ input bandwidth, no dead time at the LHC, and data taking at up to 10.2 Gigasamples per second. The waveform TDC is a new concept that can reconstruct a signal by very fast sampling. Inside SAMPIC, the timing measurement is performed in three step hierarchy: (i) the coarse one using a Timestamp Gray Counter (6 ns step), (ii) followed by a medium one when the DLL is locked on the clock to define the region of interest (150 ps step) and (iii) the fine one where sampling is done in the region of interest (few ps resolution). To test the ultimate resolution of SAMPIC in ideal condition, an extensive testing campaign has been carried out on the chip itself. Fig. 6] describes the results on the time difference resolution difference as a function of the signal amplitude obtained splitting one signal into two, and delaying one by 4.73 ns. After calibration, we obtain a resolution on the time difference better 


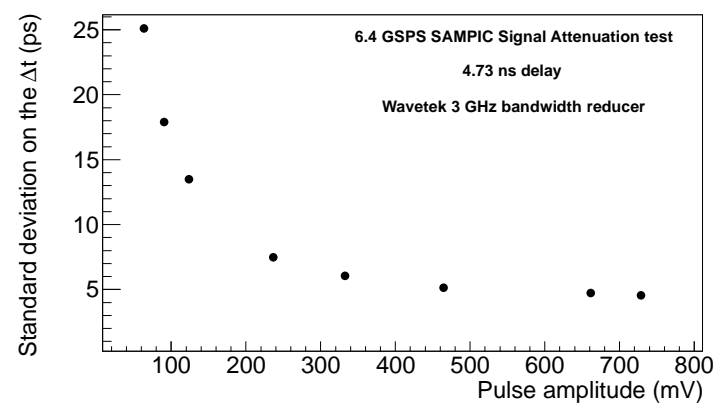

Fig. 6. Resolution of the SAMPIC chip. The resolution on the time difference between two electronic channels was measured to be about 5 ps for a signal amplitude of $500 \mathrm{mV}$ or higher, which means about 3 ps resolution per channel.

than 5 ps, which means a resolution per channel of about 3 ps for a signal amplitude larger than about $500 \mathrm{mV}$. The parameters of the SAMPIC chip are given in Fig. 7 .

SAMPIC has also been tested in combination with a new type of silicon detectors, the so called Ultra-Fast Silicon Detectors [14, that employ internal multiplication to achieve a larger signal, well suited for timing applications. Employing a split $1064 \mathrm{~nm}$ laser beam, tuned to reproduce the signal amplitude of a minimum ionizing particle, we illuminated pairs of sensors and computed, using SAMPIC, the jitter of each sensor. Fig. 8 shows the result of this test: a promising resolution of 30 ps per channel. The parameters of the SAMPIC chip are given in Fig. 7 .

Beam tests are foreseen towards the Fall 2014 to further test SAMPIC together with a diamond and $\mathrm{Si}$ detector. Further improvement of the SAMPIC chip will include the dead time reduction using the ping-pong method.

The cost per channel is estimated to be of the order for $\$ 10$ which a considerable improvement to the present cost of a few $\$ 1000$ per channel, allowing us to use this chip in medical applications such as PET imaging detectors. The holy grail of imaging 10 picosecond PET detector seems now to be feasible: with a resolution better than $20 \mathrm{ps}$, image reconstruction is no longer necessary and real-time image formation becomes possible.

The PPS project has now been endorsed by the CMS and TOTEM collaboration at least for the first phase at low luminosity. If everythings works as expected, and the beam induced background (not easily simulated) is not found to be an issue at $14 \mathrm{TeV}$, the project will be naturally approved to work at higher luminosity. The AFP project is almost at the same stage, pending the approval at low luminosities until enough resources are found 


\begin{tabular}{|c|c|c|}
\hline & & Unit \\
\hline Technology & AMS CMOS $0.18 \mu \mathrm{m}$ & \\
\hline Number of channels & 16 & \\
\hline Power consumption & 180 (1.8V supply) & $\mathrm{mW}$ \\
\hline Discriminator noise & 2 & $\mathrm{mV}$ rms \\
\hline SCA depth & 64 & Cells \\
\hline Sampling Speed & $<3-8.4$ (10.2 for 8 channels only) & GSPS \\
\hline Bandwidth & 1.6 & $\mathrm{GHz}$ \\
\hline Range (Unipolar) & 1 & $\mathrm{~V}$ \\
\hline ADC resolution & 8 to 11 (trade-off time/resolution) & bit \\
\hline SCA noise & $<1.3$ & $\mathrm{mV} \mathrm{rms}$ \\
\hline Dynamic range & 9.6 & Bit rms \\
\hline Conversion time & $0.2-1.6$ (8bit-11bit) & $\mu \mathrm{s}$ \\
\hline Readout time (can be probably be $/ 2$ ) & $25+6.2 /$ sample & ns \\
\hline Time precision before correction & 15 & ps rms \\
\hline Time precision after timing INL correction & $<5$ & ps rms \\
\hline
\end{tabular}

Fig. 7. Parameters of the SAMPIC chip.

within and outside the ATLAS collaboration.

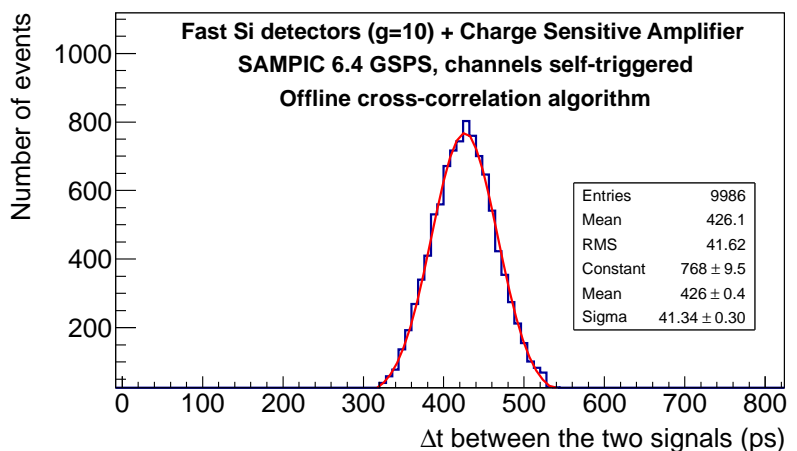

Fig. 8. Preliminary results on timing resolution using SAMPIC and a Si detector using a laser.

\section{Timimg detector optimisation for pile up rejection}

In this section, we discuss possible optimisation of the timing detector in terms of spacial resolution in order to reject pile up background. 


\subsection{Proton detection in the forward region}

The main source of background in the timing detectors is due to pile up events. Intact protons may obviously originate from the diffractive and photon-exchange events but also from additional soft interactions (pile up). For instance a non-diffractive $W W$ event can be superimposed with two single diffractive soft events with intact porons and it is important to be able to distinguish this background from the event where both protons originate from the $W W$ vertex. In order to suppress this background, it is useful to measure precisely the proton time-of-flights in order to know if the protons originate from the main event hard vertex or not.

Two parameters to build a detector are important to reject pile up:

- the precision of the proton time-of-flight, which is the timing detector resolution. Typically a measurement of 10 ps gives a precision of 2.1 $\mathrm{mm}$ on the vertex position

- the pixelisation of the timing detector: at highest luminosity, the number of intact protons per bunch crossing is high and in order to compute the time-of-flight of each proton it is needed to have enough pixelisation or space resolution so that each proton can be detected in different cells of the timing detector. If two protons with different time-of-flight fall in the same cell, the information is lost.

\subsection{Pixelisation of the timing detector}

In order to study the required pixelisation of the timing detector, we simulated 10 million minimum bias events (non diffractive, single diffractive and double diffractive events) using the PYTHIA generator. The protons were transported through the LHC magnets up to the proton detectors. Events are characterised as no tagged (NT), single tagged (ST) and double tagged (DT) depending on the number of protons in the forward proton detector acceptance. For one minimim bias event, we get a probability of $97 \% \mathrm{NT}, p=1.6 \% \mathrm{ST}$, and $q=0.01 \% \mathrm{DT}$. The multinomial distribution was adopted to simulate pile up since we assume that the different interactions are independant [15]. For a given number of pile up proton $N$, the probability to have $N_{L}\left(N_{R}\right)$ protons tagged in the left (right) side only, $N_{B}$ protons on both sides and $N_{N}$ protons not tagged reads:

$$
P\left(N_{B}, N_{L}, N_{R}, N_{N}\right)=\frac{N !}{N_{B} ! N_{L} ! N_{R} ! N_{N} !} p^{N_{L}} q^{N_{B}} p^{N_{R}}(1-2 p-q)^{N_{N}}
$$

and the probability of no proton tagged, of at least one proton tagged on the left side, and of at least one proton tagged on both sides reads

$$
P_{n o \text { hit }}=P(0,0,0, N)=(1-2 p-q)^{N}
$$




$$
\begin{aligned}
P_{\text {hit left }} & =\sum_{N_{L}=1}^{N} P\left(0, N_{L}, 0, N-N_{L}\right)=(1-p-q)^{N}-(1-2 p-q)^{N}(3) \\
P_{\text {double hit }} & =1-P_{\text {no hit }}-2 P_{\text {hit left }}=1+(1-2 p-q)^{N}-2(1-p-q)(4)
\end{aligned}
$$

\begin{tabular}{|c||c|c|c|c|}
\hline$\mu$ & $P_{N}$ & $P_{S, \text { left }}$ & $P_{S, \text { right }}$ & $P_{D}$ \\
\hline 0 & 0.97 & 0.016 & 0.016 & $9.9 \mathrm{e}-05$ \\
\hline 50 & 0.189 & - & 0.248 & 0.316 \\
\hline 100 & 0.036 & - & 0.155 & 0.655 \\
\hline 300 & 0. & - & 0.007 & 0.986 \\
\hline
\end{tabular}

Fig. 9. No tag, single tag on the left or right side (same by definition) and double tagged probablity.

The hit probabilities can then be calculated for various pile up values (see Fig. 9). For a pile up $\mu=50$ (100) for instance, the probability of no tag is $19 \%(3.6 \%)$. Let us note that this simplified approach does not work at very high pile up (300 for instance) since we neglected the cases when two or more protons from pile up events can hit one side of the detector at the same time. In order to illustrate this, the percentage of events corresponding to $0,1,2$, $3 \ldots$ protons on one side for $\mu=50,100$ and 300 is given in Fig. 10. This leads to larger inefficiencies that can be taken account in a more refined approach or by a full pile up simulation, which was performed for the $\gamma \gamma \gamma \gamma$ quartic anomalous coupling study. The detector needed to detect intact protons has a coverage of about $2 \mathrm{~cm} \times 2 \mathrm{~cm}$ and is located $15 \sigma$ from the beam. The inefficiency of such a detector assuming $20 \times 8$ pixels is given in Fig. 11. The numbers displayed in the table correspond to the probability of getting one proton or more in a given pixel for $\mu=100$ or a $20 \times 8$ pixellised detector. The upper limit on the inefficiencies if the order of $8 \%$ for the pixels closest to the bins, but is found negligible for pixels further away which measure higher mass diffractive objects. For comparison, the inefficiences for $\mu=50$ is about half, and vertical bar detectors lead to larger inefficiencies between 10 and $20 \%$ on a large part of the detector (a 7 bar detector with $2 \mathrm{~mm}$ width for the first bar and $3.25 \mathrm{~mm}$ for other bars, leads to inefficiences betweem $8 \%$ and $19 \%$ for the first 6 bars). It is also worth mentioning that this study only includes physics backgrounds and not beam-induced backgrounds which are not in the simulation. Recent results from TOTEM show that the beam-induced backgrounds have the tendency to be high and located in the pixels closest to the beam [16], and this is why a full pixelised detector is preferable to bars. 


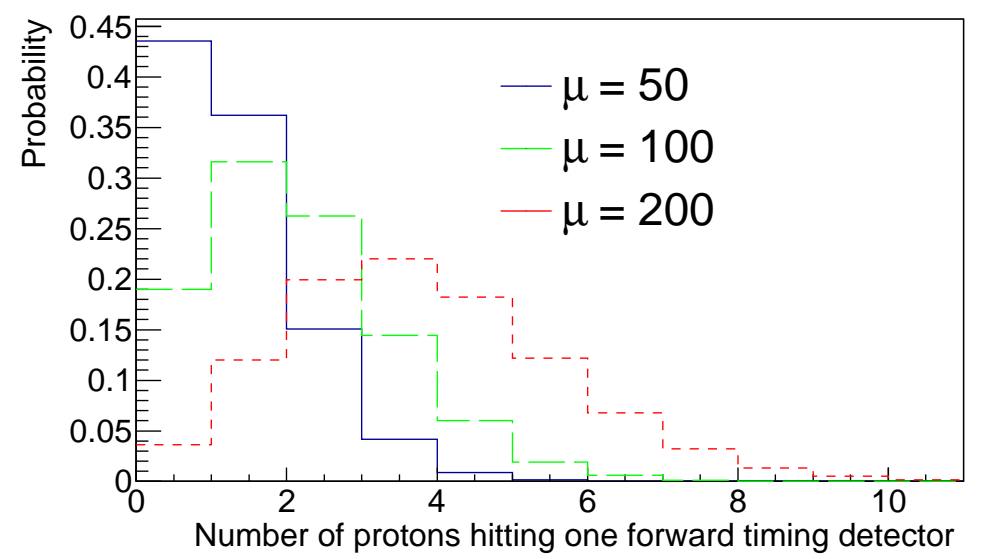

Fig. 10. Probability of getting $0,1,2,3 \ldots$ intact protons on one side of the detector for 3 different values of $\mu$.

\begin{tabular}{|c||c|c|c|c|c|c|c|c|c|c|}
\hline \multicolumn{19}{|c|}{ Inefficiencies - 20x8 pixel design $-\mu=100$} \\
\hline Row/Column & 1 & 2 & 3 & 4 & 5 & 6 & 7 & 8 & 9 & 10 \\
\hline 8 & 0. & 0. & 0. & 0. & 0. & 0. & 0. & 0. & 0. & 0. \\
\hline 7 & 0. & 0. & 0. & 0. & 0. & 0. & 0. & 0. & 0. & 0. \\
\hline 6 & 0.038 & 0.002 & 0. & 0. & 0. & 0. & 0. & 0. & 0. & 0. \\
\hline 5 & 0.067 & 0.077 & 0.051 & 0.006 & 0.001 & 0.001 & 0.001 & 0.001 & 0.001 & 0. \\
\hline 4 & 0.001 & 0.001 & 0.015 & 0.049 & 0.042 & 0.024 & 0.012 & 0.006 & 0.004 & 0.003 \\
\hline 3 & 0. & 0. & 0. & 0.001 & 0.007 & 0.019 & 0.022 & 0.018 & 0.013 & 0.008 \\
\hline 2 & 0. & 0. & 0. & 0. & 0. & 0.002 & 0.006 & 0.010 & 0.012 & 0.011 \\
\hline 1 & 0. & 0. & 0. & 0. & 0. & 0. & 0.001 & 0.003 & 0.005 & 0.007 \\
\hline \hline Row/Column & 11 & 12 & 13 & 14 & 15 & 16 & 17 & 18 & 19 & 20 \\
\hline 8 & 0. & 0. & 0. & 0. & 0. & 0. & 0. & 0. & 0. & 0. \\
\hline 7 & 0. & 0. & 0. & 0. & 0. & 0. & 0. & 0. & 0. & 0. \\
\hline 6 & 0. & 0. & 0. & 0. & 0. & 0. & 0. & 0. & 0. & 0. \\
\hline 5 & 0.001 & 0.001 & 0.001 & 0.001 & 0.001 & 0. & 0. & 0. & 0. & 0. \\
\hline 4 & 0.002 & 0.002 & 0.001 & 0.001 & 0.001 & 0.001 & 0.001 & 0.001 & 0.001 & 0. \\
\hline 3 & 0.006 & 0.004 & 0.003 & 0.003 & 0.002 & 0.002 & 0.001 & 0.001 & 0.001 & 0. \\
\hline 2 & 0.010 & 0.008 & 0.006 & 0.004 & 0.004 & 0.003 & 0.003 & 0.002 & 0.002 & 0. \\
\hline 1 & 0.008 & 0.008 & 0.007 & 0.006 & 0.005 & 0.004 & 0.004 & 0.003 & 0.003 & 0. \\
\hline
\end{tabular}

Fig. 11. Probability of more than 1 proton to fall in a given pixel of the timing detector.

\section{Acknowledgments}

The study of the timing detector optimisation was performed in collaboration with Matthias Saimpert, Oldřich Kepka and Radek Zlebčik.

\section{REFERENCES}


[1] C, Marquet, C. Royon, M. Saimpert, D. Werder, arXiv:1306.4901, Phys. Rev. D88 (2013) 7, 074029; C. Marquet, C. Royon, M. Trzebinski, R. Zlebcik, Phys.Rev. D87 (2013) 3, 034010; O. Kepka, C. Marquet, C. Royon, Phys. Rev. D83 (2011) 034036. LHC Forward Physics working group, see http://lpcc.web.cern.ch/lpcc/index.php?page=fwd_wg;

[2] E. Chapon, O. Kepka, C. Royon, Phys. Rev. D81 (2010) 074003; O. Kepka and C. Royon, Phys. Rev. D 78 (2008) 073005; S. Fichet, G. von Gersdorff, O. Kepka, B. Lenzi, C. Royon, M. Saimpert, Phys. Rev. D89 (2014) 114004.

[3] ATLAS Coll., CERN-LHCC-2011-012; M.Albrow et al., CMS-TOTEM Precision Proton Spectrometer, CERN-LHCC-2014-021.

[4] K. Piotrzkowski, U. Schneekloth, Proc. of the ZEUS Collaboration meeting, March 1994, DESY, Hamburg.

[5] ATLAS IBL Coll., CERN-LHCC-2010-013, ATLAS-TDR-019 5/09/2010.

[6] H. C. Kastli, Nucl. Instr. and Meth. A731 (2013) 88.

[7] CMS Coll., CMS Technical Design Report for the Pixel Detector Upgrade, CMS Collaboration, CERN-LHCC- 2012-016 ; CMS-TDR-11, 7 September 2012 (2012).

[8] A. Brandt, Microchannel Plate PMT Lifetime and Performance, DIRC2011: Workshop on Fast Cerenkov detectors, Giessen, Germany, April 4-6, 2011.

[9] CMS Coll., CMS Technical Design Report for the Phase 1 Upgrade of the Hadron Calorimeter, Technical Re- port CERN-LHCC-2012-015. CMS-TDR010, CERN, Geneva, (Sep, 2012).

[10] N. Cartiglia, Proceedings of the workshop on timing detectors, ClermontFerrand, March 12-14 2014.

[11] G. Chiodini, Proceedings of the workshop on timing detectors, ClermontFerrand, March 12-14 2014.

[12] C. Royon, M. Saimpert, this report.

[13] E. Delagnes, D. Breton, F. Lugiez, R. Rahmanifard, Nucl. Science IEEE Transactions, Volume 54, Issue 5, Part 2, Oct 2007, 1735; E. Delagnes, patent WO 2008/050177. EP2076963; E. Delagnes, Proceedings of the workshop on timing detectors, Clermont-Ferrand, March 12-14 2014; H. Grabas, PhD thesis, University of Paris Sud, December 2013.

[14] N. Cartiglia et al 2014 JINST 9 C02001. doi:10.1088/1748-0221/9/02/C02001

[15] M. Saimpert, master thesis, Ecole des Mines de Paris (2014).

[16] M. Beretti, talk given at the "LHC Working Group on Forward Physics and Diffraction", 14-18 April 2014, Trento, Italy. 